\title{
Smoking cessation: health system challenges and opportunities
}

\author{
Andrew L Pipe ำ, ${ }^{1}$ William Evans, ${ }^{2}$ Sophia Papadakis ${ }^{3}$
}

'Division of Cardiac Prevention and Rehabilitation, University of Ottawa Heart Institute, Ottawa, Ontario, Canada

${ }^{2}$ Department of Oncology, McMaster University Faculty of Health Sciences, Hamilton, Ontario, Canada

${ }^{3}$ Clinic of Social and Family Medicine, University of Crete School of Medicine, Heraklion, Crete, Greece

\section{Correspondence to}

Dr Andrew L Pipe, Division of Prevention and Rehabilitation, University of Ottawa Heart Institute, Ottawa, ON K1Y 4W7, Canada; apipe@ottawaheart.ca

Received 30 June 2021 Accepted 24 January 2022

\section{Check for updates}

(c) Author(s) (or their employer(s)) 2022. No commercial re-use. See rights and permissions. Published by BMJ.

To cite: Pipe AL, Evans W, Papadakis S. Tob Control 2022:31:340-347.

\section{ABSTRACT}

The systematic integration of evidence-based tobacco treatment has yet to be broadly viewed as a standardof-care. The Framework Convention on Tobacco Control recommends the provision of support for tobacco cessation. We argue that the provision of smoking cessation services in clinical settings is a fundamental clinical responsibility and permits the opportunity to more effectively assist with cessation. The role of clinicians in prioritising smoking cessation is essential in all settings. Clinical benefits of implementing cessation services in hospital settings have been recognised for three decades - but have not been consistently provided. The Ottawa Model for Smoking Cessation has used an 'organisational change' approach to its introduction and has served as the basis for the introduction of cessation programmes in hospital and primary care settings in Canada and elsewhere. The significance of smoking cessation dwarfs that of many preventive interventions in primary care. Compelling evidence attests to the importance of providing cessation services as part of cancer treatment, but implementation of such programmes has been slow. We recognise that the provision of such services must reflect the realities and resources of a particular health system. In low-income and middle-income countries, access to treatment facilities pose unique challenges. The integration of cessation programmes with tuberculosis control services may offer opportunities; and standardisation of peri-operative care to include smoking cessation may not require additional resources. Mobile phones afford unique opportunities for interactive cessation programming. Health system change is fundamental to improving the provision of cessation services; clinicians can be powerful advocates for such change.

\section{INTRODUCTION}

Smoking cessation is arguably the most powerful, cost-effective intervention available in clinical settings for the primary and secondary prevention of disease, disability and death. ${ }^{1-4}$ The continued carnage, and enormous costs, that result from global tobacco addiction make tobacco control and smoking cessation fundamental public-health priorities. ${ }^{6}$ That one-sixth of all non-communicable disease deaths can be attributed to smoking, and that smokers consume a markedly disproportionate share of expensive healthcare resources underscore the importance of robust approaches to the treatment of tobacco addiction in clinical facilities. Notwithstanding such evidence, the systematic integration of evidence-based tobacco treatment interventions in clinical settings-both hospital and primary care-has yet to be widely viewed as a standard-of-care. We affirm that the provision of smoking cessation services in clinical settings is a fundamental clinical responsibility and affords the opportunity to more efficiently and effectively assist with cessation. It is important to recognise that our experience and perspectives reflect practice in hospital and primary care settings in a high-income nation. Regrettably, despite considerable progress, the delivery of cessation services in clinical settings in our own communities is far from universal.

We acknowledge that the provision of smoking cessation services must be addressed in a manner commensurate with available resources and the structure of a particular health system.

The Framework Convention on Tobacco Control (FCTC) - a legally-binding, multilateral treaty signed by 182 countries-has significantly impacted tobacco control since its introduction in $2005 .^{7}$ An important element of the FCTC, Article 14 , recommends the provision of evidence-based support for tobacco cessation. Progress in implementing Article 14 has been slow. ${ }^{8}$ WHO reports regularly on progress in implementing the elements of the FCTC by assessing progress in six activities (Monitoring tobacco use, Protecting, Offering cessation assistance, Warning of tobacco's dangers, Enforcement of advertising bans and Raising taxes (MPOWER)). In its most recent report, WHO notes that 'the number of countries adopting comprehensive tobacco-cessation measures lags behind the other MPOWER measures'. 9 To be successful in addressing this challenge requires 'implementing policies that transform systems of care to better address tobacco use and dependence, promoting evidence-based treatments for tobacco cessation and implementing policies that are clinically focused and promote cessation'. ${ }^{3}$ Government support and the influence of accreditation agencies can play fundamental roles in stimulating and supporting the delivery of such services in clinical settings. Such leadership notwithstanding, it has been noted that the introduction of standards and accreditation criteria require their adoption and implementation by hospitals and clinical facilities-adoption that is not always forthcoming. ${ }^{10}$ We recognise that the model of health system present in any nation will influence the scope and style of cessation services that can be provided. The FCTC affords an opportunity for Member States to implement smoking- cessation services at all levels of their health systems. In low-income and middle-income (LMIC) countries, access to treatment facilities and medications pose unique challenges. Developing solutions specific to the needs of LMICs 'is a key to effective tobacco control'. ${ }^{1112}$ An understanding of the cultural factors that motivate 
cessation is essential to enhance the development and efficacy of cessation programmes. ${ }^{13}$ In many settings, the integration of smoking cessation programmes with tuberculosis control services affords specific opportunity. ${ }^{14}$ The need 'especially in LMICs to place a greater spotlight on smoking cessation after a cancer diagnosis' has been emphasised. ${ }^{15}$ Standardisation of peri-operative care to include smoking cessation is feasible 'even with limited resources'. ${ }^{16}$ The widespread availability of mobile phones in LMICs affords unique opportunities to support cessation and connectivity to other cessation resources (mCessation) but evidence of their effectiveness is unclear and merits further study. ${ }^{17}$

The role of clinicians in making a priority of smoking cessation has been recognised as central to ending the tobacco pandemic ${ }^{18}$; the need and opportunity for systematic approaches to the delivery of smoking-cessation services in clinical environments have frequently been identified. ${ }^{319-22}$ Estimates of the substantial impact the introduction of such approaches would have on rates of hospitalisation, clinical outcomes and healthcare spending have been published. ${ }^{23}{ }^{24}$ Yet the identification and documentation of smoking status, and the provision of cessation assistance in clinical settings are often more serendipitous than systematic. Ironically, the conspicuous absence of 'smoking cessation' as an element of the clinical-treatment guidelines for many smokingrelated diseases has recently been noted. ${ }^{25}$ Lack of training and lack of time are often reported by clinical staff as impediments to the delivery of smoking- cessation treatment, ${ }^{26}{ }^{27}$ and the cost of providing cessation services is often cited as an obstacle by administrators despite evidence of the cost-effectiveness of such important clinical programmes. ${ }^{28} 29$ Opportunities abound within hospital, primary care and specialty care settings to ensure the delivery of systematic, evidence-based cessation programmes. ${ }^{20}$ If we are to advance in the tobacco 'End Game', optimisation of cessation services is essential; and clinicians can play an important role by advocating for their introduction.

\section{SMOKING CESSATION IN HOSPITAL SETTINGS}

Systematic approaches to cessation in hospital settings were recognised as providing important clinical benefits 30 years ago. ${ }^{30} 31$ Such recognition notwithstanding, evidence-based smoking cessation treatments are not consistently provided in hospital settings-prompting one author to wonder when hospitals will 'do the right thing'? ${ }^{32}$ Illness can be a powerful motivation to quit. The provision of cessation medication in hospital, accompanied by bedside support can forestall the discomfort of nicotine withdrawal while permitting the initiation of a smoking cessation attempt, and ongoing support can be provided following discharge. ${ }^{33-35}$ There is substantial evidence regarding the efficacy of hospital-based cessation interventions in reducing rates of smoking in both the short term and long term. ${ }^{36}$ Almost 50 years ago, it was noted that hospitalisation following myocardial infarction afforded a unique opportunity to successfully assist with smoking cessation. ${ }^{37}$ The substantial role that tobacco addiction plays in cardiac morbidity and mortality has been recognised for decades. ${ }^{38}$ Multiple investigators have identified the very significant clinical benefits that follow the delivery of smoking cessation in cardiac settings. ${ }^{39-45}$ Moreover, the advantages of hospital smoking cessation programmes have been clearly documented across an array of clinical disciplines: addressing smoking among surgical patients ${ }^{46-48}$; combating peri-operative complications by anaesthesiologists ${ }^{49}{ }^{50}$; reducing the multiple smoking-related complications following orthopaedic procedures ${ }^{51-57}$; demonstrating the benefits of cessation services in psychiatric settings ${ }^{58-60}$ and identifying opportunities to deliver tobacco-dependence treatment in emergency departments. ${ }^{6162}$

\section{THE OTTAWA MODEL FOR SMOKING CESSATION}

It has been recognised that the successful introduction of a smoking cessation programme in hospital settings requires a concerted, well-organised effort to ensure the integration of the elements necessary for a viable and effective clinical service. ${ }^{63}$ The Ottawa Model for Smoking Cessation (OMSC) developed at the University of Ottawa Heart Institute, successfully used an 'organisational change' approach to its introduction, and has since become the basis of multiple successful cessation programmes in Canada and elsewhere. ${ }^{406465}$ The OMSC has been designed using knowledge, principles and 'best practices' drawn from the fields of tobacco control, implementation science, quality improvement and organisational change. The model ensures the identification and documentation of the smoking status of all admitted patients, prompts an offer of smoking cessation assistance using evidence-based best practice, provides for the follow-up of patients attempting cessation postdischarge and has demonstrated high long-term cessation rates. ${ }^{64}$ A robust evaluation of the OMSC in 14 Ontario (Canada) hospitals showed a substantial reduction in 30-day, 1-year and 2-year healthcare usage as reflected in reduced rates of readmission and emergency department visits following implementation. ${ }^{23}$ The OMSC has also been demonstrated to be cost-effective from the hospital-payer perspective with low intervention costs in contrast to the costs of readmissions for smoking-related illnesses. ${ }^{24}$ The adoption (and effectiveness) of models like the OMSC can be enhanced when healthcare funders introduce performance expectations regarding smoking cessation in funding agreements with hospitals and other clinical providers. ${ }^{66}$

\section{SMOKING CESSATION IN PRIMARY CARE AND GENERAL PRACTICE SETTINGS}

Numerous international clinical practice guidelines have identified primary- care settings as important sites for cessation assistance. ${ }^{267-72}$ The rates at which simple advice to quit smoking is delivered by primary-care practitioners vary widely. ${ }^{73-75} 7576$ The rates at which actual assistance with cessation using evidencebased treatment, including pharmacotherapy and counselling, is delivered are suboptimal in most settings. ${ }^{374476-79}$

The impact of successful smoking cessation dwarfs that of many other commonly provided preventive interventions in primary care (table 1). ${ }^{12}$ However, many clinicians find it challenging to deliver evidence-based tobacco treatment in busy primary-care settings. The majority have not received training in how to support the delivery of evidence-based tobacco treatments to their patients. ${ }^{80}$ Other barriers limiting the delivery of tobacco treatment in primary care include: a lack of time; lack of confidence in addressing cessation with patients; outdated attitudes regarding the nature of tobacco addiction and the role of primary care in addressing tobacco use; lack of practice supports to identify smokers and the absence of effective performance incentives (box 1). ${ }^{81-85}$

There is good evidence demonstrating that training primarycare clinicians in smoking-cessation delivery increases the rate of tobacco treatment. ${ }^{86}{ }^{87}$ There is emerging evidence that introducing smoking-cessation protocols that are supported by electronic medical-record 'prompts' and templates increase rate of tobacco-treatment delivery in primary care ${ }^{88}{ }^{89}$ Interventions that facilitate immediate, easy access to affordable 


\begin{tabular}{|c|c|}
\hline Intervention & NNT \\
\hline \multicolumn{2}{|l|}{ Smoking cessation } \\
\hline Brief advice & 40 \\
\hline \multicolumn{2}{|l|}{ Intensive behavioural support with } \\
\hline Nicotine Replacement Therapy (NRT) & $23 * / 46 t$ \\
\hline Varenicline & $10 * / 20 t$ \\
\hline Statins as primary prevention & 107 \\
\hline Antihypertension treatment for mild hypertension & 700 \\
\hline Screening for cervical cancer & 1140 \\
\hline Mammography & 205 \\
\hline Papanicolaou smear & 534 \\
\hline Pneumococcal vaccine & 716 \\
\hline
\end{tabular}

Modified from: Critchley and Capewell'; Van Schayck et al. ${ }^{2}$

*NNT to have one person quit smoking.

tNNT to prevent one death.

(ideally cost-free) pharmacotherapy and behavioural support from others in a practice setting (usually practice nurses or a community-based cessation service) have been found to be effective in increasing rates of cessation among smokers in primary care settings. 8687

While evidence concerning the role of provider incentives and performance feedback when delivered as stand-alone strategies is unclear, performance measurement is key to identifying practice gaps and supporting quality improvement. ${ }^{71868790}$ The most promising strategies for increasing performance in the delivery of tobacco-dependence treatment in primary care settings are multicomponent interventions designed to address the multiple barriers that impede treatment delivery. ${ }^{7186879091}$

\section{THE OTTAWA MODEL FOR SMOKING CESSATION IN PRIMARY CARE}

The OMSC has been adapted for use in primary care practices and has been implemented in $>200$ group-based primary care practices in Ontario (Canada). The OMSC in primary care settings emphasises systematic, interdisciplinary collaboration permitting busy, primary care clinics to adopt the programme while enhancing both efficiency and effectiveness. Three evaluations of the OMSC in primary care settings have documented a significant increase in primary care provider delivery of evidence-based tobacco-dependence treatments. ${ }^{739293}$ A pre-post evaluation of the first 32 family health teams (481 providers) in Ontario to implement the model found that the rate of delivery of the 3 As (Ask, Advise, Act) increased significantly following programme implementation (figure 1). ${ }^{92}$

Box 1 Barriers to tobacco treatment delivery in primary care

Patient's motivation to quit and resistance.

Lack of clinician knowledge and skills.

Time constraints.

Smoking not considered a primary care priority.

Complexity of the intervention.

Lack of practice supports.

Clinician attitudes, beliefs, self-efficacy and practice norms.

Lack of incentives.

Lack of accountability.

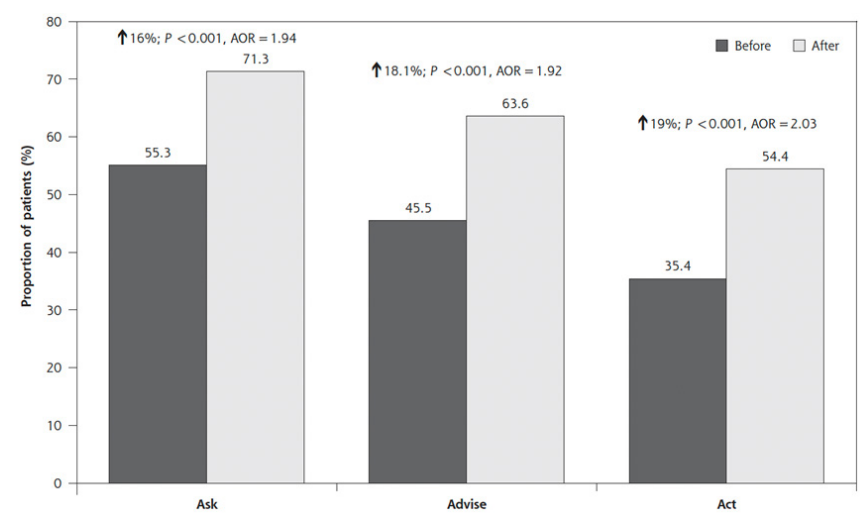

Figure 1 Clinic performance in the 3 As delivery before and after implementation of OMSC. Note: The AORs presented control for cliniclevel and provider-level variance between clusters, availability of costfree nicotine replacement therapy, gender of the patient, self-reported time of first cigarette and purpose of visit; based on inclusion of 32 clinics and 481 providers. P values are based on the Wald statistic. 3 As, Ask, Advise, Act; AOR, adusted OR; OMSC, Ottawa Model for Smoking Cessation.

The Ottawa Model adapted for primary care includes two key elements specifically applicable in such practice settings: (1) an evidence-based tobacco treatment protocol that guides intervention delivery to patients using the 3 As model (table 2) and (2) practice-change supports to facilitate its high-quality implementation in healthcare organisations (figure 2). A suite of implementation supports is introduced to assist clinics with systematising tobacco treatment (table 3). These include training in the development of an evidence-based smoking cessation protocol; training for frontline staff (physicians, nurses, allied health professionals) in evidence-based tobacco treatment and the OMSC protocol; the provision of customised practice tools and resources (standardised documentation, medical directives, patient educational materials); electronic medical-record integration (which includes measurement capability); digital and telephone follow-up scripts and materials to complement faceto-face contact and, importantly, materials to support performance evaluation. Ongoing evaluation, and modification of the programme to meet the specific needs and circumstances of a particular practice setting, ensure that cessation services remain optimised.

\section{CESSATION IN CANCER TREATMENT SETTINGS}

The evidence that continued smoking after a diagnosis of cancer results in worse clinical outcomes is compelling. ${ }^{349495}$ Smoking increases all-cause mortality, leads to greater treatment-related toxicity, reduces the efficacy of radiation and some systemic therapies and increases the incidence of second malignancies. Smoking cessation in oncology centres provides significant advantage to the patient with cancer. The 2014 US Surgeon General's report estimates that if patients stopped smoking after a cancer diagnosis cancer mortality could be reduced by $30 \%-40 \%$. $^{34}$

Although the harms of continued smoking and the potential benefits of stopping have become better known in the oncology community, implementation of smoking cessation initiatives within cancer centres has been slow. Implementation has been impeded by myths that smokers would not be receptive to advice about quitting and concerns that raising the issue of cessation would undermine the relationship between the oncologist and 
Table 2 The OMSC in primary care-Ask, Advise, Act model

\begin{tabular}{|c|c|c|}
\hline Clinical practice & Responsible primary care team member(s) & Description \\
\hline $\begin{array}{l}\text { Ask } \\
(30 \mathrm{~s})\end{array}$ & Triage nurse/Medical assistant & $\begin{array}{l}\text { Ask and document } \\
\text { Include tobacco use question as one of the patient's vital signs: "Have you used any form of tobacco in } \\
\text { the last } 7 \text { days?" "Have you used any form of tobacco in the past?" }\end{array}$ \\
\hline $\begin{array}{l}\text { Advise } \\
\text { (2 min) }\end{array}$ & General practitioner/Nurse practitioner & $\begin{array}{l}\text { Advise, offer support and refer } \\
\text { Provide strong, personalised, non-judgmental advice to quit with offer of support. } \\
\text { Arrange rapid referral to smoking cessation counsellor. } \\
\text { Provide brief, periodic, motivational intervention to persons not ready to quit. }\end{array}$ \\
\hline $\begin{array}{l}\text { Act } \\
(20-30 \mathrm{~min})\end{array}$ & $\begin{array}{l}\text { Smoking cessation counsellor (eg, nurse, nurse } \\
\text { practitioner, pharmacist) }\end{array}$ & $\begin{array}{l}\text { For patient who is ready to quit } \\
\text { Quit plan visit: } \\
\text { Strategic counselling; } \\
\text { Pharmacotherapy; } \\
\text { Follow-up ( } 2-6 \text { months). } \\
\text { Stient who is not ready to quit } \\
\text { Sollowic counselling (reduce to quit/smoking reduction) pharmacotherapy; } \\
\text { Self-help material. }\end{array}$ \\
\hline
\end{tabular}

OMSC, Ottawa Model for Smoking Cessation.

their patient, especially if the issue is raised at the first consultation visit. ${ }^{96}$ Oncologists often indicate that they lack training in smoking cessation pharmacotherapy and in how to effectively counsel persons who use tobacco. ${ }^{95}$ A common refrain is that they are just too busy to address smoking cessation given the increasing complexity of the cancer care they are required to direct. In contrast, there is clear evidence that training oncologycare providers can have demonstrable impact on improving tobacco-treatment skills and tobacco-treatment delivery. ${ }^{97}$

If oncologists are to achieve the best clinical outcomes for their patients, the oncology centres in which they work must implement strategies to ensure that all newly diagnosed patients with cancer are screened for smoking status, advised of the benefits of smoking cessation and referred to smoking cessation services. An 'opt out' approach to the provision of smoking-cessation care can distinctly enhance the ability to ensure patients receive the supports required to optimise their cancer care. ${ }^{98}$ Furthermore, all patients with cancer should continue to have their smoking status monitored after the completion of treatment. Those who continue to use tobacco should be repeatedly encouraged to stop in order to reduce the risk of recurrence, second primaries and non-oncological tobacco-related illnesses, such as cardiovascular and chronic lung diseases.
Patients with cancer do expect their oncologists to ask about their smoking status. ${ }^{99}$ In a survey of 501 cancer survivors at the Princess Margaret Hospital (Toronto, Canada), 96\% were comfortable with being assessed and $87 \%$ felt that smoking cessation discussions should occur at the first visit. Patients have little or no idea of the negative impacts of continued smoking on cancer treatment outcomes-yet another reason why oncology care providers need to educate them on the importance of quitting before cancer treatment. ${ }^{100}$

In 2012, Cancer Care Ontario, which at the time was responsible for managing the province's cancer services, introduced a framework for the implementation of smoking cessation in its 14 regional cancer centres. ${ }^{101}$ The elements of the framework included screening all new patients with cancer for their smoking status using standardised screening questions, provision of advice on the health benefits of smoking cessation and referral to smoking- cessation supports. Initially, referral was simply recommended but subsequently an 'opt out' approach was adopted and resulted in an increased proportion of individuals receiving cessation support. ${ }^{102}$ All cancer centres were required to identify a smoking-cessation champion and to commit to staff support and training. Cancer Care Ontario provided central administrative support. Regions determined that the intensity and location

\begin{tabular}{lcc}
\hline & $\begin{array}{c}\text { Before } \\
\text { Intervention }\end{array}$ & $\begin{array}{c}\text { After } \\
\text { Intervention }\end{array}$ \\
\hline Clinics with the OMSC 10 Best Practices implemented, by best practice, \% & 3.1 & 93.8 \\
1. Clinic task force formed & 0.0 & 96.9 \\
2. Clinic tobacco control protocol developed & 28.1 & 81.3 \\
3. Tobacco use queried and documented for all clinic patients & 28.1 & 90.6 \\
4. Training in tobacco dependence treatment offered to health care providers in last year & 59.4 & 96.9 \\
5. Staff trained and available to provide tobacco dependence treatment & 68.8 & 90.6 \\
6. Self-help materials readily available to patients, family members, and staff & 3.1 & 90.6 \\
7a. EMR or other real time prompt in place to inform clinician of patient smoking status & 43.8 & 93.8 \\
7b. EMR supports in place (smoking status identification, consult form, etc) & 3.1 & 93.8 \\
8. Process to follow-up tobacco users for at least 1 month after clinic visit in place & 3.1 & 71.9 \\
9. Process to evaluate quality or program implementation in place & 2.4 & 81.3 \\
10. Process to provide feedback to clinicians about performance in place & 0.0 & 8.9 \\
Average number of best practices implemented, No. & 59.3 \\
Clinics with 10 best practices implemented, \% & & \\
\hline
\end{tabular}

Figure 2 Ten best practices for tobacco treatment delivery in primary care before and after implementation of OMSC in 32 family health teams. Based on data from 32 primary care practices. EMR, electronic medical record; OMSC, ottawa model for smoking cessation. 


\begin{tabular}{|c|c|}
\hline Intervention components & Description \\
\hline Outreach facilitation visits & $\begin{array}{l}\text { A trained outreach facilitator worked with each primary care clinic over a 3-month period to implement the programme. } \\
\text { - } \text { - review current clinic proctices in the delivery of evidence-based smoking-cessation intervention and complete needs assessment; } \\
\text { - provide information and recommendations on the integration of evidence-based smoking-cessation strategies into clinical practice; } \\
\text { - facilitate development of a clinic tobacco treatment protocol for integrating evidence-based smoking cessation strategies into all } \\
\text { clinic appointments; } \\
\text { - define roles and responsibilities of clinic staff in delivering evidence-based smoking-cessation treatments; } \\
\text { - support communications and training activities for members of the clinic staff. }\end{array}$ \\
\hline $\begin{array}{l}\text { Standardised provider and patient } \\
\text { tools }\end{array}$ & $\begin{array}{l}\text { All materials were designed to support intervention delivery and reduce the amount of face-to-face time required to support tobacco } \\
\text { treatment delivery. These included: } \\
\text { Patient Tobacco Use Survey to document smoking history. } \\
\text { Checklist style Smoking Cessation Consult Form. } \\
\text { Patient Quit Plan booklet for Smokers Ready to Quit. } \\
\text { Booklet for Smokers Not Ready to Quit. } \\
\text { Clinic waiting room posters and materials. }\end{array}$ \\
\hline $\begin{array}{l}\text { Adjunct follow-up support and } \\
\text { counselling }\end{array}$ & $\begin{array}{l}\text { Patient's ready to quit could be referred to the telephone-based Smoker's Follow-up System, which included five triage calls over a 2-month } \\
\text { period delivered by Interactive Voice Response System. Patients struggling with their quit attempt received additional telephone-based } \\
\text { support from trained smoking-cessation counsellors. }\end{array}$ \\
\hline Coaching and feedback session & $\begin{array}{l}\text { A supplemental } 1.5 \text {-hour group-based coaching session was delivered approximately } 4 \text { weeks following the launch of the OMSC at their } \\
\text { clinic. This was facilitated by a trained tobacco treatment specialist using a standardised facilitation guide. The performance coaching } \\
\text { session was designed to increase provider self-efficacy in tobacco treatment delivery, identify personal barriers to tobacco treatment } \\
\text { delivery and exchange experiences in addressing these barriers. }\end{array}$ \\
\hline
\end{tabular}

of the interventions offered reflected available resources. Some centres relied exclusively on a provincial quit-line while others used in-house smoking cessation counsellors, hospital pharmacists and social workers or a combination of internal and external resources. Critical to the success of implementation was seniorlevel commitment and ongoing education of healthcare workers and patients regarding the importance of cessation. Monitoring of performance drove provincial screening rates above $70 \%$ and referral rates of smokers to $30 \%$.

The COVID-19 pandemic spurred the use of virtual care and the capture of smoking status through phone or video patient registration and consultation. It also accelerated the deployment of provider and patient smoking- cessation learning materials and the development, at the Princess Margaret Hospital, of digital education prescriptions that direct patients to online smoking- cessation learning resources. ${ }^{103}$

The Ontario initiative has spread across Canada through the leadership and funding of the Canadian Partnership Against Cancer and now, virtually all cancer centres screen patients with cancer for their smoking status and direct current smokers to cessation resources. ${ }^{104} 105$ The Cancer Center Cessation Initiative (C3I), part of the National Cancer Institute Cancer Moonshot programme, has similarly increased the spread of smoking cessation initiatives within major US cancer centres. ${ }^{106} 107$ In 2017, 22 cancer centres received 2 years of funding to implement smoking cessation programmes and in 2018 an additional 20 centres joined the initiative. C3I is designed to achieve a broad clinical impact by identifying patients with cancer who smoke, providing them with advice to quit, offering evidence-based cessation treatment and assessing treatment outcomes. C3I will help to identify how evidence-based tobacco interventions can best be introduced into clinical practice in cancer-treatment settings.
Despite the excellent progress in Canada and the USA, challenges remain in both countries and across the globe. It is essential that the culture within all cancer-treament facilities changes to make smoking cessation an integral part of high-quality cancer treatment and care. Further progress would no doubt be accelerated in Canada by the introduction by Accreditation Canada of cancer-centre standards requiring screening of patients with cancer for their smoking status, the provision of advice on the health benefits of cessation and the referral of persons who use tobacco to smoking-cessation services. The implementation of such standards and public reporting of performance on these metrics would drive change and lead to better clinical outcomes for patients with cancer.

\section{CONCLUSION}

Health system change is fundamental if we are to effectively assist the large number of tobacco-dependent patients who present for care in various clinical facilities. ${ }^{108}$ Notwithstanding the growing evidence of the effectiveness of smoking cessation programmes in a wide range of clinical settings and the demonstrable need for such services, it has been difficult to persuade administrators and healthcare funders of their value. Smoking has been mischaracterised by many as a 'habit' or a 'lifestyle choice' and there has been an indifference to the circumstances and needs of smokers. The cost of services is often cited as an obstacle to their introduction-despite evidence of the savings provided by cessation programmes. ${ }^{23}$ Those attitudes are outdated and inaccurate. Funding agencies and governments can clearly influence the development and delivery of these services by defining programme expectations as a condition of funding. ${ }^{66}$ Innovative approaches for ensuring the integration of smoking 
What this paper adds

- A cogent description of the need for the systematic integration of smoking-cessation services in clinical settings.

- Evidence of the effectiveness of an 'organisational change' approach to the introduction of such services in hospital and other facilities.

- Reinforcement of the need to deliver smoking-cessation services in primary-care settings.

- Identification of the Ottawa Model for Smoking Cessation as a useful prototype for the implementation of cessation services.

- Affirmation of the powerful clinical impact of smokingcessation services in oncology settings.

- Recognition of the role of the Framework Convention on Tobacco Control in galvanising the delivery of cessation services and the need for novel approaches to the delivery of smoking-cessation services in low-income and middle-income countries.

cessation into clinical protocols and treatment algorithms have been described. ${ }^{36} 109$ Clinicians can be powerful advocates for the establishment of such services and accreditation agencies can require that smoking cessation is seen as a contemporary 'standard-of-care'. But success will not be achieved without the commitment of senior administrators and clinical leaders, the development of team approaches to intervention delivery and a recognition of the need to integrate tobacco-related measures with quality-improvement processes.

In assessing the impact of tobacco dependence, it has been noted that 'it is difficult to identify any other condition that presents such a mix of lethality, prevalence and neglect, despite effective and readily available interventions' ${ }^{19} 66$ If we are to successfully address the tobacco pandemic in clinical settings, we must end the years of neglect by adopting best practices and, implementing systematic approaches to the identification and treatment of smokers.

Contributors All authors have contributed equally to the preparation and review of this manuscript.

Funding The authors have not declared a specific grant for this research from any funding agency in the public, commercial or not-for-profit sectors.

Competing interests None declared.

Patient consent for publication Not applicable.

Ethics approval This study does not involve human participants.

Provenance and peer review Commissioned; externally peer reviewed.

ORCID iD

Andrew L Pipe http://orcid.org/0000-0003-1280-1801

\section{REFERENCES}

1 Critchley JA, Capewell S. Mortality risk reduction associated with smoking cessation in patients with coronary heart disease: a systematic review. JAMA 2003;290:86-97.

2 Van Schayck OCP, Williams S, Barchilon V, et al. Treating tobacco dependence: guidance for primary care on life-saving interventions. position statement of the IPCRG. NPJ Prim Care Respir Med 2017;27:38.

3 United States Public Health Service Office of the Surgeon General. Smoking cessation: a report of the surgeon General. Washington (DC): National Center for Chronic Disease Prevention and Health Promotion (US) Office on Smoking and Health, 2020

4 National Center for Chronic Disease Prevention and Health Promotion. The Health Consequences of Smoking - 50 Years of Progress: A Report of the Surgeon General. Atlanta (GA): Centers for Disease Control and Prevention (US), 2014.
5 GBD 2019 Tobacco Collaborators. Spatial, temporal, and demographic patterns in prevalence of smoking tobacco use and attributable disease burden in 204 countries and territories, 1990-2019: a systematic analysis from the global burden of disease study 2019. Lancet 2021:397:2337-2360.

6 Jha P, Ramasundarahettige C, Landsman V, et al. 21St-Century hazards of smoking and benefits of cessation in the United States. N Engl J Med 2013;368:341-50.

7 Organization. WH. Who framework convention on tobacco control. Geneva: World Health Organization, 2005.

8 Raw M, Ayo-Yusuf 0, Chaloupka F, et al. Recommendations for the implementation of who framework convention on tobacco control article 14 on tobacco cessation support. Addiction 2017;112:1703-8.

9 Organization. WH. Who report on the global tobacco epidemic 2021: addressing new and emerging products. Geneva: World Health Organization, 2021.

10 Fiore MC, Goplerud E, Schroeder SA. The Joint Commission's new tobacco-cessation measures--will hospitals do the right thing? N Engl J Med 2012;366:1172-4.

11 Kumar N, Janmohamed K, Jiang J, et al. Tobacco cessation in low- to middleincome countries: a scoping review of randomized controlled trials. Addict Behav 2021;112:106612.

12 Iqbal S, Barolia R, Ladak L, et al. Smoking cessation interventions in South Asian countries: protocol for scoping review. BMJ Open 2021;11:e038818.

13 Martins RS, Junaid MU, Khan MS, et al. Factors motivating smoking cessation: a cross-sectional study in a lower-middle-income country. BMC Public Health 2021:21:1419.

14 Goel S, Siddiqi K, Singh RJ, et al. Fuelling the tuberculosis epidemic: the role of tobacco control in ending the TB emergency. Indian J Tuberc 2019;66:555-60.

15 Golčić M, Tomaš I, Stevanović A, et al. Smoking cessation after a cancer diagnosis: a cross-sectional analysis in the setting of a developing country. Clin Pract 2021;11:509-19.

16 Abdel Jalil R, Al-Najjar H, Abou Chaar MK, et al. The impact of a perioperative pulmonary care bundle implementation on postoperative outcomes in 1,665 surgical cancer patients: experience from a tertiary referral cancer center in Jordan. Patient Saf Surg 2021;15:5

17 Krishnan N, Gu J, Abroms LC. Mobile phone-based messaging for tobacco cessation in low and middle-income countries: a systematic review. Addict Behav 2021:113:106676.

18 Blum A, Eke R. Tobacco control: all research, no action. Lancet 2021

19 Fiore MC, Jaen CR, Baker TB. Treating tobacco use and dependence: 2008 update. clinical practice guideline. Rockville, MD: US Dept of Health and Human Services. Public Health Service, 2008.

20 National Institute for Clinical Excellence (NICE). Smoking: acute, maternity and mental health services (PH48). London: NICE, 2013.

21 Royal College of Physicians. Hiding in plain sight: treating tobacco dependency in the NHS. London: RCP, 2018.

22 Zoorob RJ, Levine RS, Hennekens CH. Smoking cessation and hospitalized patients: a missed opportunity to avoid premature deaths. Ochsner J 2021;21:10-13.

23 Mullen KA, Manuel DG, Hawken SJ, et al. Effectiveness of a hospital-initiated smoking cessation programme: 2-year health and healthcare outcomes. Tob Control 2017:26:293-9

24 Mullen K-A, Coyle D, Manuel D, et al. Economic evaluation of a hospital-initiated intervention for smokers with chronic disease, in Ontario, Canada. Tob Control 2015;24:489-96

25 Ekezie W, Murray RL, Agrawal S, et al. Quality of smoking cessation advice in guidelines of tobacco-related diseases: an updated systematic review. Clin Med 2020;20:551-9.

26 Russell L, Whiffen R, Chapman L, et al. Hospital staff perspectives on the provision of smoking cessation care: a qualitative description study. BMJ Open 2021:11:e044489.

27 Raupach T, Merker J, Hasenfuss G, et al. Knowledge gaps about smoking cessation in hospitalized patients and their doctors. Eur J Cardiovasc Prev Rehabil 2011;18:334-41

28 Maciosek MV, Coffield AB, Edwards NM, et al. Priorities among effective clinical preventive services: results of a systematic review and analysis. Am J Prev Med 2006;31:52-61.

29 Levy DE, Klinger EV, Linder JA, et al. Cost-Effectiveness of a health System-Based smoking cessation program. Nicotine Tob Res 2017;19:1508-15.

30 Glasgow RE, Stevens VJ, Vogt TM, et al. Changes in smoking associated with hospitalization: quit rates, predictive variables, and intervention implications. Am J Health Promot 1991;6:24-9.

31 Levy DE, Chang Y, Regan S, et al. Improvements in health-related quality of life among smokers who quit after hospitalization. Prev Med 2018;110:38-46.

32 Fiore MC, Adsit R. Will Hospitals Finally "Do the Right Thing"? Providing EvidenceBased Tobacco Dependence Treatments to Hospitalized Patients Who Smoke. Jt Comm J Qual Patient Saf 2016;42:207-8.

33 Rigotti NA, Arnsten JH, McKool KM, et al. Efficacy of a smoking cessation program for hospital patients. Arch Intern Med 1997;157:2653-60.

34 Evison M, Pearse C, Howle F, et al. Feasibility, uptake and impact of a hospital-wide tobacco addiction treatment pathway: results from the cure project pilot. Clin Med 2020;20:196-202. 
35 Rigotti NA, Clair C, Munafò MR, et al. Interventions for smoking cessation in hospitalised patients. Cochrane Database Syst Rev 2012;5:Cd001837.

36 Rigotti NA. li. smoking cessation in the hospital setting-a new opportunity for managed care. Introduction. Tob Control 2000;9 Suppl 1:154-5.

37 Burt A, Thornley $P$, Illingworth $D$, et al. Stopping smoking after myocardial infarction. Lancet 1974;1:304-6.

38 Kannel WB, Doyle JT, McNamara PM, et al. Precursors of sudden coronary death. factors related to the incidence of sudden death. Circulation 1975;51:606-13.

39 Thomson CC, Rigotti NA. Hospital- and clinic-based smoking cessation interventions for smokers with cardiovascular disease. Prog Cardiovasc Dis 2003;45:459-79.

40 Reid RD, Pipe AL, Quinlan B. Promoting smoking cessation during hospitalization for coronary artery disease. Can J Cardiol 2006;22:775-80

41 Mohiuddin SM, Mooss AN, Hunter CB, et al. Intensive smoking cessation intervention reduces mortality in high-risk smokers with cardiovascular disease. Chest 2007;131:446-52.

42 Van Spall HGC, Chong A, Tu JV. Inpatient smoking-cessation counseling and all-cause mortality in patients with acute myocardial infarction. Am Heart J 2007:154:213-20.

43 Dawood N, Vaccarino V, Reid KJ, et al. Predictors of smoking cessation after a myocardial infarction: the role of institutional smoking cessation programs in improving success. Arch Intern Med 2008;168:1961-7.

44 Reid RD, Mullen KA, Pipe AL. Systematic approaches to smoking cessation in the cardiac setting. Curr Opin Cardiol 2011;26:443-8.

45 Samaan Z, Nowacki B, Schulze K, et al. Smoking cessation intervention in a cardiovascular hospital based clinical setting. Cardiovasc Psychiatry Neurol 2012;2012:970108

46 Howard R, Singh K, Englesbe M. Prevalence and trends in smoking among surgical patients in Michigan, 2012-2019. JAMA Netw Open 2021;4:e210553.

47 Creager MA, Matsushita K, Arya S, et al. Reducing Nontraumatic Lower-Extremity Amputations by $20 \%$ by 2030 : Time to Get to Our Feet: A Policy Statement From the American Heart Association. Circulation 2021;143:e875-91.

48 Garip M, Van Dessel J, Grosjean L, et al. The impact of smoking on surgical complications after head and neck reconstructive surgery with a free vascularised tissue flap: a systematic review and meta-analysis. Br J Oral Maxillofac Surg 2021;59:e79-98.

49 Grønkiær M, Eliasen M, Skov-Ettrup LS, et al. Preoperative smoking status and postoperative complications: a systematic review and meta-analysis. Ann Surg 2014:259:52-71.

50 Lee SM. Perioperative smoking cessation programs should be standard-of-care. Can J Anaesth 2019:66:849-50.

51 Singh JA, Schleck C, Harmsen WS, et al. Current tobacco use is associated with higher rates of implant revision and deep infection after total hip or knee arthroplasty: a prospective cohort study. BMC Med 2015;13:283.

52 Jackson KL, Devine JG. The effects of smoking and smoking cessation on spine surgery: a systematic review of the literature. Global Spine J 2016;6:695-701.

53 Rodriguez-Merchan EC. The importance of smoking in orthopedic surgery. Hosp Pract 2018;46:175-82.

54 Halawi MJ, Allen DA, Baron S, et al. Tobacco smoking independently predicts lower patient-reported outcomes: new insights on a forgotten epidemic. J Arthroplasty 2019;34:S144-7.

55 Herrero C, Tang A, Wasterlain A, et al. Smoking cessation correlates with a decrease in infection rates following total joint arthroplasty. J Orthop 2020;21:390-4.

56 Agrawal S, Ingrande J, Said ET, et al. The association of preoperative smoking with postoperative outcomes in patients undergoing total hip arthroplasty. J Arthroplasty 2021;36:1029-34.

57 Badiee RK, Chan AK, Rivera J, et al. Smoking is an independent risk factor for 90-day readmission and reoperation following posterior cervical decompression and fusion. Neurosurgery 2021;88:1088-94.

58 Prochaska JJ, Hall SE, Delucchi K, et al. Efficacy of initiating tobacco dependence treatment in inpatient psychiatry: a randomized controlled trial. Am J Public Health 2014; 104:1557-65

59 Brown RA, Minami H, Hecht J, et al. Sustained care smoking cessation intervention for individuals hospitalized for psychiatric disorders: the helping hand 3 randomized clinical trial. JAMA Psychiatry 2021;78:839-847.

60 McKelvey K, Thrul J, Ramo D. Impact of quitting smoking and smoking cessation treatment on substance use outcomes: an updated and narrative review. Addict Behav 2017:65:161-70

61 Bernstein SL, D'Onofrio G, Rosner J, et al. Successful tobacco dependence treatment in low-income emergency department patients: a randomized trial. Ann Emerg Med 2015:66:140-7.

62 Tolmie AD, Erker R, Oyedokun T, et al. Prevalence of cigarette smoking among adult emergency department patients in Canada. West J Emerg Med 2020;21:190-7.

63 Reid RD, Mullen K-A, Pipe AL. Tackling smoking cessation systematically among inpatients with heart disease. CMAJ 2018;190:E345-6.

64 Reid RD, Mullen K-A, Slovinec D'Angelo ME, D'Angelo ME, et al. Smoking cessation for hospitalized smokers: an evaluation of the "Ottawa Model". Nicotine Tob Res 2010;12:11-18.
65 Evison M, Agrawal S, Conroy M, et al. Building the case for comprehensive hospital-based tobacco addiction services: applying the Ottawa model to the city of Manchester. Lung Cancer 2018;121:99-100.

66 Mullen KA, Walker KL, Hobler LA, et al. Performance obligations to improve delivery of Hospital-Initiated smoking cessation interventions: a before-and-after evaluation. Nicotine Tob Res 2021:23:77-84.

67 West R, McNeill A, Raw M. Smoking cessation guidelines for health professionals: an update. health education authority. Thorax 2000:55:987-99.

68 (ENSP) ENfSP. Guidelines for treating tobacco dependence. Brussels, ENSP, 2017 Brussels, 2017.

69 Zwar NRR, Borland R, Peters M, et al. Supporting smoking cessation: a guide for health professionals. Melbourne, 2011.

70 Patnode CD, Henderson JT, Coppola EL, et al. Interventions for tobacco cessation in adults, including pregnant persons: updated evidence report and systematic review for the US preventive services Task force. JAMA 2021;325:280-98.

71 Fiore MC JC, Baker TB, Bailey WC, et al. Treating tobacco use and dependence: 2008 update. clinical practice guideline, 2008.

72 Verbiest M, Brakema E, van der Kleij R, et al. National guidelines for smoking cessation in primary care: a literature review and evidence analysis. NPJ Prim Care Respir Med 2017;27:2

73 Papadakis S, McDonald PW, Pipe AL, et al. Effectiveness of telephone-based follow-up support delivered in combination with a multi-component smoking cessation intervention in family practice: a cluster-randomized trial. Prev Med 2013;56:390-7

74 Bartsch A-L, Härter M, Niedrich J, et al. A systematic literature review of selfreported smoking cessation counseling by primary care physicians. PLoS One 2016;11:e0168482.

75 Organization. WH. Who report on the global tobacco epidemic 2019: offer help to quit tobacco use. Geneva, 2019

76 Jamal A, Dube SR, Malarcher AM, et al. Tobacco use screening and counseling during physician office visits among adults--National Ambulatory Medical Care Survey and National Health Interview Survey, United States, 2005-2009. MMWR Supp/ 2012:61:38-45

77 Kruger J, O'Halloran A, Rosenthal A. Assessment of compliance with U.S. public health service clinical practice guideline for tobacco by primary care physicians. Harm Reduct J 2015;12:7

78 Stead LF, Koilpillai P, Fanshawe TR, et al. Combined pharmacotherapy and behavioural interventions for smoking cessation. Cochrane Database Syst Rev 2016:3:CD008286.

79 Papadakis S, Gharib M, Hambleton J, et al. Delivering evidence-based smoking cessation treatment in primary care practice: experience of Ontario family health teams. Can Fam Physician 2014;60:e362-71.

80 Raupach T, Shahab L, Baetzing S, et al. Medical students lack basic knowledge about smoking: findings from two European medical schools. Nicotine Tob Res 2009;11:92-8.

81 Vogt F, Hall S, Marteau TM. General practitioners' and family physicians' negative beliefs and attitudes towards discussing smoking cessation with patients: a systematic review. Addiction 2005:100:1423-31.

82 Young JM, Ward JE. Implementing guidelines for smoking cessation advice in Australian general practice: opinions, current practices, readiness to change and perceived barriers. Fam Pract 2001;18:14-20.

83 Vaughn TE, Ward MM, Doebbeling BN, et al. Organizational and provider characteristics fostering smoking cessation practice guideline adherence: an empirical look. J Ambul Care Manage 2002;25:17-31.

84 van Rossem C, Spigt MG, Kleijsen JRC, et al. Smoking cessation in primary care: exploration of barriers and solutions in current daily practice from the perspective of smokers and healthcare professionals. Eur J Gen Pract 2015;21:111-7.

85 Martin-Cantera C, Sanmartín JMI, Martínez AF, et al. Good practice regarding smoking cessation management in Spain: challenges and opportunities for primary care physicians and nurses. Tob Prev Cessat 2020;6:55.

86 Anderson P, Jané-Llopis E. How can we increase the involvement of primary health care in the treatment of tobacco dependence? A meta-analysis. Addiction 2004;99:299-312.

87 Papadakis S, McDonald P, Mullen K-A, et al. Strategies to increase the delivery of smoking cessation treatments in primary care settings: a systematic review and meta-analysis. Prev Med 2010;51:199-213.

88 Bae J, Ford EW, Huerta TR. The electronic medical record's role in support of smoking cessation activities. Nicotine Tob Res 2016;18:1019-24.

89 Bae J, Ford EW, Kharrazi HHK, et al. Electronic medical record reminders and smoking cessation activities in primary care. Addict Behav 2018:77:203-9.

90 Martín Cantera C, Puigdomènech E, Ballvé JL, et al. Effectiveness of multicomponent interventions in primary healthcare settings to promote continuous smoking cessation in adults: a systematic review. BMJ Open 2015;5:e008807.

91 Lindson N, Pritchard G, Hong B, Fanshawe B.;, et al. Strategies to improve smoking cessation rates in primary care. Cochrane Database Syst Rev 2021;9:CD011556.

92 Papadakis S, Cole AG, Reid RD, et al. Increasing rates of tobacco treatment delivery in primary care practice: evaluation of the Ottawa model for smoking cessation. Ann Fam Med 2016;14:235-43. 
93 Papadakis S, Cole AG, Reid RD, et al. From good to great: the role of performance coaching in enhancing Tobacco-Dependence treatment rates. Ann Fam Med 2018;16:498-506.

94 Toll BA, Brandon TH, Gritz ER, et al. Assessing tobacco use by cancer patients and facilitating cessation: an American association for cancer research policy statement. Clin Cancer Res 2013;19:1941-8.

95 Warren GW, Marshall JR, Cummings KM, et al. Practice patterns and perceptions of thoracic oncology providers on tobacco use and cessation in cancer patients. $J$ Thorac Oncol 2013;8:543-8.

96 Giuliani M, Brual J, Cameron E, et al. Smoking cessation in cancer care: myths, presumptions and implications for practice. Clin Oncol 2020;32:400-6.

97 Ostroff JS, Bolutayo Gaffney KL, O'Brien M, et al. Training oncology care providers in the assessment and treatment of tobacco use and dependence. Cancer 2021;127:3010-3018.

98 Himelfarb-Blyth S, Vanderwater C, Hartwick J. Implementing a 3As and 'Opt-Out' Tobacco Cessation Framework in an Outpatient Oncology Setting. Curr Oncol 2021;28:1197-203.

99 L LS E, Zhang Q, et al. Cancer patient attitudes and preferences towards smoking status assessment. J Clin Oncol 2018;36:177-77.

100 Eng L, Alton D, Song Y, et al. Awareness of the harms of continued smoking among cancer survivors. Support Care Cancer 2020;28:3409-19.
101 Evans WK, Truscott R, Cameron E, et al. Lessons learned implementing a province-wide smoking cessation initiative in Ontario's cancer centres. Curr Oncol 2017;24:185-90.

102 Evans WK, Truscott R, Cameron E, et al. Implementing smoking cessation within cancer treatment centres and potential economic impacts. Trans/ Lung Cancer Res 2019:8:S11-20.

103 Giuliani M, Papadakos T, Papadakos J. Propelling a new era of patient education into Practice-Cancer care Post-COVID-19. Int J Radiat Oncol Biol Phys 2020:108:404-6.

104 Halligan M, Keen D, Timmings C, Warren GW, et al. Results of a pan-Canadian approach to systems change for smoking cessation support in Canadian cancer centers. JCO 2018;36:e18538.

105 Canadian Partnership Against Cancer. Leading practices in smoking cessation programs 2020, 2020.

106 Croyle RT, Morgan GD, Fiore MC. Addressing a Core Gap in Cancer Care - The $\mathrm{NCl}$ Moonshot Program to Help Oncology Patients Stop Smoking. N Engl J Med 2019;380:512-5.

107 D'Angelo H, Rolland B, Adsit R, et al. Tobacco treatment program implementation at $\mathrm{NCl}$ cancer centers: progress of the $\mathrm{NCl}$ cancer Moonshot-Funded cancer center cessation initiative. Cancer Prev Res 2019:12:735-40.

108 Fiore MC, Keller PA, Curry SJ. Health system changes to facilitate the delivery of tobacco-dependence treatment. Am J Prev Med 2007;33:S349-56.

109 The agency for health care policy and research smoking cessation clinical practice guideline. JAMA 1996;275:1270-80. 